\title{
Can We Prevent or Delay Type 2 Diabetes?
}

\author{
Merita Emini Sadiku
}

Additional information is available at the end of the chapter

http://dx.doi.org/10.5772/54872

\section{Introduction}

Type 2 diabetes results from the interaction between genetic predisposition, behavioral and environmental risk factors (1). There is strong evidence that modifiable risk factors such as obesity and physical inactivity are the main non-genetic determinants of the disease(2). New figures indicate that the number of people living with diabetes is expected to rise from 366 million to 552 million by 2030, if no urgent action is taken (3).

Impaired glucose tolerance (IGT) is an intermediate category between normal glucose tolerance and overt diabetes, and it can be identified by glucose tolerance test. Subjects with IGT have an increased risk of type 2 diabetes (4), and consequently many trials of interventions for the prevention of type 2 diabetes have focused on such individuals. By 2025 , the number of people with IGT is projected to increase to 418 million, or $8.1 \%$ of the adult population (5).

Interventions to delay or even to prevent type 2 diabetes have a potential to improve the health of the population and reduce the burden of healthcare costs. The interventions assessed have been diverse and include pharmacological and lifestyle changes.

Abnormal glucose tolerance is also a well-known risk factor of incident diabetes (6). The main known predictor of incident diabetes is the presence of insulin resistance. Direct measures of insulin sensitivity can be laborious, complicated, and expensive to be applicable for routine use in the general population as a screening tool. Markers like HOMA-IR index or 2-hour post-challenge glucose levels are being used to diagnose insulin resistance.

IGT when compared to impaired fasting glucose (IFG) is a stronger risk factor in predicting the onset of diabetes (7). Diagnosing of IGT requires oral glucose tolerance test (OGTT), a test of high specificity (92\%) but low sensitivity (52\%) in the prediction of diabetes (8). The World Health Organization (WHO) criteria including either IFG $(\geq 6.1 \mathrm{mmol} / \mathrm{L}$ and $<7.0$ $\mathrm{mmol} / \mathrm{L}$ and 2-h plasma glucose concentration $<11.1 \mathrm{mmol} / \mathrm{L}$ during the oral glucose 
tolerance test) or IGT test $(<7.0 \mathrm{mmol} / \mathrm{L}$ and 2 -h plasma glucose concentration $\geq 7.8 \mathrm{mmol} / \mathrm{L}$ and $<11.1 \mathrm{mmol} / \mathrm{L}$ ) are used to diagnose people with IGT (9).

The clinical characteristics associated with type 2 diabetes risk include obesity and overweight, age (the risk rises steadily from puberty into geriatric years), a history of gestational diabetes, polycystic ovary syndrome, a family history of type 2 diabetes, and membership in certain high-risk minority groups: African American, Hispanic, Native American, and Asian-Pacific Islanders (6). The ADA recommends screening youth and adults with multiple risk factors for type 2 diabetes; fasting plasma glucose is the preferred first-line test $(10,11)$.

People with metabolic syndrome are at a high risk to develop diabetes and ischemic atherosclerotic diseases (7). Several studies have shown that metabolic syndrome is a strong predictor of incident diabetes (12-14).

The Insulin Resistance Atherosclerosis Study (IRAS) assessed a series of risk factors and identified five significant risk factors for the development of type 2 diabetes including high plasminogen activator inhibitor 1 (PAI-1), hypertension (HTN), high triglycerides (TG), low high-density lipoprotein (HDL), and IGT. The incidence of type 2 diabetes increases with the increasing number of risk factors, $5 \%$ with 0 risk factors to $50 \%$ when all 5 risk factors are present $(p<0.001)(15)$.

Every year about $5 \%-10 \%$ of people with IGT will develop diabetes and acquire the disease burden related to its diagnosis symptoms, need for surveillance for chronic consequences and associated costs, and increased risk of several chronic diseases (16).

The purpose of this article is to review available evidence on lifestyle and pharmacological intervention on the prevention or delay of the onset of type 2 diabetes and adapting these lessons from clinical trials to clinical practice.

\section{How we can prevent type 2 diabetes?}

The high economic and social costs of type 2 diabetes mellitus and its rising prevalence make a compelling case for its prevention (17). The most vulnerable groups to have diabetes are people with IFG and IGT and in the mean time they present the potent groups of preventing or delaying the progression to type 2 diabetes mellitus. These two groups are recognized as prediabetic states by the ADA (11).

\section{Non pharmacological intervention}

Four large prospective studies demonstrated that a treatment regimen using diet plus exercise reduces IGT progression to type 2 diabetes (18-21). Factor analysis showed that both insulin resistance and insulin secretion had a significant association with the development of diabetes, and lifestyle intervention was more effective in subjects with lower insulin resistance and higher insulin secretion (13). 


\subsection{Lifestyle trials - diet and physical activity}

Overweight, obesity and physical inactivity are the major risk factors for developing type 2 diabetes mainly through insulin resistance. Therefore, interventions in weight reduction were shown to enhance insulin sensitivity and improve glucose tolerance in nondiabetic and diabetic subjects.

In the DaQing study, 577 subjects with IGT as defined by the WHO criteria were followed over 6 years. They were randomized into diet-only, exercise only, diet plus exercise groups, and a control group, which were associated with a 31\% ( $p \leq 0.03), 46 \%(p \leq 0.0005)$ and $42 \%$ $(p \leq 0.005)$ reduction in the risk of developing diabetes, respectively, when compared to the control group. Factor analysis showed that both insulin resistance and insulin secretion had a significant association with the development of diabetes, and lifestyle intervention was more effective in subjects with lower insulin resistance and higher insulin secretion $(15,21,22)$.

The Swedish Diabetes Prevention Study was the first individually randomized clinical trial, where 522 middle-aged overweight subjects (body mass index/BMI >25) with IGT were randomized to the control group or intervention group and followed over a mean of 3.2 years. The intervention group received individual counseling regarding diet and exercise and lost $4.2 \pm 5.1 \mathrm{~kg}$ versus $0.8 \pm 3.7 \mathrm{~kg}$ in the control group, which translated to a $58 \%(p \leq 0.001)$ reduction in the risk of developing diabetes and was directly associated with changes in lifestyle $(18,23)$.

The Diabetes Prevention Program (DPP) study was a double-blind randomized controlled trial (RCT) involving a larger number of subjects, 3234 with IGT or IFG with BMI $>24 \mathrm{~kg} / \mathrm{m} 2$ ( $>22$ in Asian population) followed over 2.8 years. They were randomized to standard lifestyle recommendations with placebo or with metformin or to an intensive program of lifestyle modifications (19). The incidence of developing diabetes was 4.8 cases versus 11 cases per 100 person years for 3 years in the intensive lifestyle and placebo groups, respectively, which was a $58 \%$ lower incidence of developing diabetes in the intensive lifestyle group. In this study these changes were observed in all of the various ethnic and racial subgroups, and at least $50 \%$ of the lifestyle group had achieved the goal of $>7 \%$ of weight reduction.

The Look AHEAD trial, which, like DPP, demonstrated impressive weight loss at year 1, was also followed by significant weight regain over the subsequent 3 years despite continued intensive intervention and follow-up (24).

In a recent community study in Finland, 2,797 high-risk individuals were enrolled in a diabetes prevention program designed to achieve $5-7 \%$ body weight loss. Only approximately one-third of participants were able to successfully decrease body weight by $2.5 \%(25)$.

From above mention studies it is obvious that successful weight reduction alone is not sufficient to prevent diabetes in a large percentage of individuals. The main issues is that it is difficult to maintain weight without an intervention program. 
The key components of lifestyle interventions in clinical practice based on the studies analyzed by Burnet et al. (37) were as follows: the staff included were medical doctors, nurses, technicians, dietitians and physiotherapists. Trainings with patients were focused on nutrition, physical activity and behavioral self-management four times during the year in individualized sessions (in Diabetes Prevention Study) or small group counseling sessions weekly for one month, then monthly for three months (32). Physical activities were organized 2 times during the week as brisk walking (in Diabetes Prevention Program and Malmo Feasibility Study). Smokers were advised to stop or reduce smoking. It was a useful follow up session every 2 months with phone calls to patients between visits. In DPS study, if weight goal was not achieved in 6 to 12 months, a very low calorie diet was considered. As for social support, spouses were invited to join sessions.

The overall goal for diabetes prevention is to reach and maintain an active, healthy weight with a tendency toward a hypocaloric diet. Evidence supports limiting total calories and fat ( $25 \%$ of caloric intake) and increasing dietary fiber (20 to $30 \mathrm{~g} /$ day). Essential skills include understanding portion sizes and reading food labels (37).

\section{Pharmacological interventions}

Weight regain is a characteristic feature of most weight reduction programs, irrespective of the type of dietary intervention and similarly, weight loss achieved with pharmacological intervention is associated with major weight regain once medication is discontinued.

As shown above, lifestyle modification is the best strategy to prevent the progression of metabolic risk factors and to prevent cardiovascular events and the onset of diabetes. However, pharmacological trials have shown important results.

\subsection{Metformin}

Metformin (MF) $850 \mathrm{mg}$ was used in one arm of the DPP study along with standard lifestyle recommendations. Subjects in this study had a decrease in their calorie intake by a mean of $296 \pm 23 \mathrm{kcal}$ compared to $249 \pm 27 \mathrm{kcal}$ in the placebo group; their average fat intake decreased by $0.8 \pm 0.2 \%$ in both the MF and the placebo group ( $p=\mathrm{NS}$ ).

The DPP demonstrated a 31\% reduction in IGT conversion to type 2 diabetes in subjects receiving metformin, $850 \mathrm{mg}$ twice daily $(15,21)$.

\section{2. $\alpha$-Glucosidase inhibitors}

Both acarbose (STOP-NIDDM) (26) and voglibose (27) have been shown to decrease conversion of IGT to type 2 diabetes. Although this preventive effect initially was believed to result from inhibition of carbohydrate absorption, $\alpha$-glucosidases augment incretin hormone secretion; thus, enhanced b-cell function could, in part, explain their beneficial effects on glucose homeostasis (28). Subjects on acarbose were by $25 \%$ less likely to develop 
diabetes compared to placebo at the end of 1 year, and this continued to the end of the study. The reduced risk was present even after adjusting for change in weight, age, sex, or BMI $(p=0.0063)(26)$.

\subsection{Thiazolidinediones}

Thiazolidinediones (TZD) are insulin sensitizers that act by facilitating glucose transport into the muscle and by acting on Perioxisome Proliferator Activated Receptor (PPAR)- $\gamma$ receptors in the adipose cells to shift fat from visceral to less active subcutaneous fat compartment thus reducing insulin resistance. The TRIPOD was a single-center, placebocontrolled randomized controlled trials, which enrolled Mexican-American women with prior gestational diabetes mellitus (GDM) randomized to either placebo or troglitazone 400 $\mathrm{mg} /$ day. The mean annual incidence of diabetes was $5.4 \%$ in the troglitazone group versus $12.1 \%$ in the placebo group ( $p=0.009$ ), which was a $>50 \%$ reduction with troglitazone use (29). Analysis done 8 months after troglitazone had been stopped showed that the mean annual incidence of diabetes was $21.2 \%$ and $3.1 \%$ in the placebo and troglitazone group, respectively. This indicated that the protection by the drug persisted even after it had been stopped. The study was continued in the same group of women using pioglitazone called the Pioglitazone In the Prevention of Diabetes (PIPOD) study and the results published indicated that the benefit in terms of $\beta$-cell function achieved with troglitazone was maintained with the use of pioglitazone, indicating that it could be a class effect (30). Also, a report appeared on diabetes reduction assessment in the Ramipril and Rosiglitazone medication (DREAM) trial (16). This trial reported only combined results for individuals with either IGT or IFG. Briefly, it was found that the ACE inhibitor ramipril did not significantly reduce the incidence of diabetes (hazard ratio $0.91,0.80$ to 1.03 ) but rosiglitazone, an oral diabetes drug, did (0.38, 0.33 to 0.44$)$.

In another study ACT NOW, pioglitazone ( $45 \mathrm{mg} /$ day) decreased by $72 \%$ ( $\mathrm{P}<0.00001)$ IGT conversion to type 2 diabetes (31).

\subsection{Orlistat}

Orlistat is another pharmacological agent used in the prevention of diabetes. Orlistat is a weight-reduction agent that inhibits the activity of intestinal lipase and thus decreases the amount of fat (triglycerides) absorbed. After 4 years' treatment, the cumulative incidence of diabetes was $9.0 \%$ with placebo and $6.2 \%$ with orlistat, corresponding to a risk reduction of $37.3 \%(\mathrm{P}=0.0032)$. Exploratory analyses indicated that the preventive effect was explained by the difference in subjects with IGT. Mean weight loss after 4 years was significantly greater with orlistat (5.8 vs. $3.0 \mathrm{~kg}$ with placebo; $\mathrm{P}<0.001$ ) and similar between orlistat recipients with impaired $(5.7 \mathrm{~kg}$ ) or normal glucose tolerance (NGT) $(5.8 \mathrm{~kg})$ at baseline. A second analysis in which the baseline weights of subjects who dropped out of the study was carried forward also demonstrated greater weight loss in the orlistat group (3.6 vs. $1.4 \mathrm{~kg}$; P $<0.001)(32)$. 


\subsection{GLP-1 analogs}

Liraglutide and exenatide are GLP-1 receptor agonists that mimic the actions of GLP-1 and are resistant to dipeptidyl peptidase-4 degradation. GLP-1 analogs represent a logical therapeutic intervention for treatment of IGT. Moreover, the stimulatory effect of GLP-1 and GLP-1 analogs on insulin secretion is glucose dependent, minimizing risk for hypoglycemia (33). Once-daily liraglutide also reduces body weight and decreases IGT conversion to type 2 diabetes in obese nondiabetic subjects (34).

\subsection{Angiotensin converting enzyme inhibitor (ACEI)/angiotensinogen receptor blocker (ARB)}

In addition to the beneficial effects on hypertension, the kidneys and the heart, both ACEI and ARBs have been shown to improve insulin sensitivity and glycemic control. Therapy with ACEI, like captopril and ramipril, and ARBs, like losartan and valsartan has been shown to reduce the incidence of new-onset diabetes anywhere by $14 \%$ to $34 \%$ (18). Although the exact mechanism how these agents reduce the incidence of diabetes is not known, it is well established that ACEI increases glucose uptake in skeletal muscle through increased synthesis of GLUT- 4 transporter protein secondary to up-regulation of insulin receptor substrate 1 (IRS 1) activity, enhanced bradykinin and nitric oxide (NO) activity (35).

\subsection{Statins}

The West of Scotland Coronary Prevention Study (WOSCOPS) examined the effect of pravastatin on cardiovascular (CV) events and observed that these pharmacological interventions were associated with a $30 \%$ reduction in the incidence of diabetes as secondary outcome (36). Therapeutic agents used to treat other coexisting conditions like hypertension and dyslipidemia with agents like ACEI/ARB/statins can also help with the prevention of diabetes and CV disease (15).

\section{Conclusions and recommendations}

From the trials discussed it was shown that both non pharmacological and pharmacological intervention can reduce the risk of type 2 diabetes in people with IGT, but lifestyle intervention seems to be more effective than pharmacological interventions. Lifestyle intervention, which aims to reduce obesity and increase physical activity, helps in addressing directly these risk factors. In the mean time lifestyle interventions incur fewer and less serious side effects than drug treatment. Like in pharmacological interventions, lifestyle interventions may not be permanent and advice on diet and exercise needs to be regularly reinforced by professional staff. For pharmacological interventions, adverse effects need to be fully understood to enable the potential harms and benefits to be assessed.

In lifestyle intervention, clinicians should recommend behavior changes for asymptomatic patients at a high risk of diabetes such as IGT. High-risk patients can be identified through 
clinical characteristics augmented with careful screening by fasting glucose. Although the diabetes prevention trials used intensive strategies for effecting lifestyle change, clinicians can translate key elements from those strategies into brief, office-based counseling on physical activity and dietary change. As it was proved by different trials in clinical practice, lifestyle changes should be made better through structured programs. These programs, proved to be successful in clinical practices from different countries, should emphasize goal setting, practice and motivational interviewing with patients, education and skills development, self-monitoring, behavior change (cognitive restructuring), physical activity, problem solving, stress and stimulus control, the importance of social support, and the utilization of community resources.

Multidisciplinary care teams consisting of nurses, clinicians, dietitians, psychologist physiotherapists and health educators may provide more intensive counseling and increase the motivation to continue and achieve the targets. Other forms of awareness rising as printed materials or if possible interactive computer programs in offices can reinforce counseling efforts. Implementing diabetes prevention will require significant changes for both patients and clinicians. Every health system that provide these special services need to educate clinicians in training and in practice about the potential benefits of diabetes prevention. Appropriate programs on the prevention or delay of type 2 diabetes have to be culturally adaptive for office-based counseling considering the better results planned to achieve. Besides office bases counseling with patients and family members, successful diabetes prevention efforts through lifestyle changes will likely require involvement broader societal entities such as schools, communities and workplaces.

\section{Author details}

Merita Emini Sadiku

University of Pristina, Kosovo

\section{References}

[1] Neel JV. Diabetes mellitus: a "thrifty" genotype rendered detrimental by "progress"? Am J Hum genet 1962;14:353-62.

[2] Manson JE, Rimm EB, Stampfer MJ, et al. Physical activity and incidence of non insulin dependent diabetes mellitus in women. Lancet 1991;338:774-8.

[3] International Diabetes Federation. Diabetes Atlas, 5rd ed. Brussels: International DiabetesFederation; 2011.

[4] The DECODE Study Group. Glucose tolerance and mortality: comparison of WHO and American Diabetes Association diagnostic criteria. Lancet 1999;354:617-21.

[5] International Diabetes Federation. Diabetes Atlas, 3rd ed. Brussels: International DiabetesFederation; 2006. 
[6] Stern MP, Williams K, Haffner SM. Identification of persons at high risk for type 2 diabetes mellitus: do we need the oral glucose tolerance test? Ann Intern Med 2002;136:575-81.

[7] Emini-Sadiku M, Car N, Metelko Z, Bajraktari G, Morina N, Devolli D. Prevention or delay of type 2 diabetes by pharmacological or lifestyle interventions. Diabetologia Croatica 2008;37-1.

[8] Orio Jr, F Palomba S, Cascella T, et al. Improvement in endothelial structure and function after metformin treatment in young normal-weight women with polycystic ovary syndrome: results of a 6-month study. J Clin Endocrinol Metab 2005;90:6072-6.

[9] World Health Organization. Definition, diagnosis and classification of diabetes mellitus and its complications: report of a WHO consultation. Part1: diagnosis and classification of diabetes mellitus.Geneva: World Health Organization; 1999.

[10] American Diabetes Association. Type 2 diabetes in children and adolescents. Diabetes Care 2000;23:381-9.

[11] American Diabetes Association. Screening for type 2 diabetes. Diabetes Care 2004; 27:S11-3.

[12] Meigs JB, Williams K, Sullivan LM, et al. Using metabolic syndrome traits for efficient detection of impaired glucose tolerance. Diabetes Care 2004;27:1417-26.

[13] Hanley AJ, Festa A, D'Agostino Jr RB, et al. Metabolic and inflammation variable clusters and prediction of type 2 diabetes: factor analysis using directly measured insulin sensitivity. Diabetes 2004;53:1773-81.

[14] Grundy SM, Brewer Jr, HB Cleeman JI, et al.Definition of metabolic syndrome: Report of the National Heart, Lung, and Blood Institute/American Heart Association conference on scientific issues related to definition. Circulation 2004;109:433-8.

[15] Babu A, Fogelfeld L. Metabolic syndrome and prediabetes. Dis Mon 2006;52 (2-3):55144.

[16] DREAM Trial Investigators. Effect of rosiglitazone on the frequency of diabetes in patients with impaired glucose tolerance or impaired fasting glucose: a randomised controlled trial. Lancet 2006; 368:1096-105.

[17] Zimmet P, Alberti KG, Shaw J. Global and societal implications of the diabetes epidemic. Nature 2001;414:782-7.

[18] Tuomilehto J, Lindstrom J, Eriksson JG, et al. Prevention of type 2 diabetes mellitus by changes in lifestyle among subjects with impaired glucose tolerance. N Engl J Med 2001;344:1343-50.

[19] Knowler WC, Barrett-Connor E, Fowler SE, et al. Reduction in the incidence of type 2 diabetes with lifestyle intervention or metformin. N Engl J Med 2002;346:393-403.

[20] Eriksson KF, Lindgärde F. Prevention of type 2 (non-insulin-dependent) diabetes mellitus by diet and physical exercise: the 6-year Malmö feasibility study. Diabetologia 1991;34:891-898

[21] Pan XR, Li GW, Hu YH, et al. Effects of diet and exercise in preventing NIDDM in people with impaired glucose tolerance: the Da Qing IGT and Diabetes Study. Diabetes Care 1997;20:537-544 
[22] Li G, Hu Y, Yang W, et al. Effects of insulin resistance and insulin secretion on the efficacy of interventions to retard development of type 2 diabetes mellitus: the DA Qing IGT and Diabetes Study. Diabetes Res Clin Pract 2002;58:193-200.

[23] Lindstrom J, Eriksson JG, Valle TT, et al. Prevention of diabetes mellitus in subjects with impaired glucose tolerance in the Finnish Diabetes Prevention Study: results from a randomized clinical trial. J Am Soc Nephrol 2003;14:S108-13.

[24] Pi-Sunyer X, Blackburn G, Brancati FL, et al. Reduction in weight and cardiovascular disease risk factors in individuals with type 2 diabetes: one-year results of the Look AHEAD trial. Diabetes Care 2007;30:1374-1383

[25] Saaristo T, Moilanen L, Korpi-Hyövälti E, et al. Lifestyle intervention for prevention of type 2 diabetes in primary health care: one-year follow-up of the Finnish National Diabetes Prevention Program (FIN-D2D). Diabetes Care 2010; 33:2146-2151

[26] Chiasson JL, Josse RG, Gomis R, Hanefeld M, Karasik A, Laakso M; STOP-NIDDM Trial Research Group. Acarbose for prevention of type 2 diabetes mellitus: the STOP-NIDDM randomised trial. Lancet 2002;359:2072-2077

[27] Kawamori R, Tajima N, Iwamoto Y, Kashiwagi A, Shimamoto K, Kaku K; Voglibose Ph3 Study Group. Voglibose for prevention of type 2 diabetes mellitus: a randomised, double-blind trial in Japanese individuals with impaired glucose tolerance. Lancet 2009;373:1607-1614.

[28] De Fronzo R, Abdul-Ghani M.Type 2 Diabetes Can Be Prevented With Early Pharmacological Intervention. Diabetes Care 34(Suppl. 2):S202-S209, 2011

[29] Buchanan TA, Xiang AH, Peters RK, et al. Preservation of pancreatic beta-cell function and prevention of type 2 diabetes by pharmacological treatment of insulin resistance in high-risk Hispanic women. Diabetes 2002;51:2796-803.

[30] Weiss R, Taksali SE, Tamborlane WV, et al. Predictors of changes in glucose tolerance status in obese youth. Diabetes Care 2005;28:902-9.

[31] De Fronzo RA, Tripathy D, Schwenke DC, et al. Pioglitazone for diabetes prevention in impaired glucose tolerance. N Engl J Med 2011;364:1104-1115

[32] Torgerson JS, Hauptman J, Boldrin MN, et al. XENical in the prevention of diabetes in obese subjects (XENDOS) study: a randomized study of orlistat as an adjunct to lifestyle changes for the prevention of type 2 diabetes in obese patients. Diabetes Care 2004;27:155-61.

[33] Triplitt C, DeFronzo RA. Exenatide: first in class incretin mimetic for the treatment of type 2 diabetes mellitus. Exp Rev Endocirnol Metab 2006;1:329-341

[34] Astrup A, Rössner S, Van Gaal L, et al. Effects of liraglutide in the treatment of obesity: a randomised, double-blind, placebo-controlled study. Lancet 2009; 374:1606-1616

[35] Kreutzfeldt J, Raasch W, Klein HH. Ramipril increases the protein level of skeletal muscle IRS- 1 and alters protein tyrosine phosphatase activity in spontaneously hypertensive rats. Naunyn Schmiedebergs Arch Pharmacol 2000;362:1-6.

[36] The WOSCOPS Study Group. West of Scotland Coronary Prevention Study: implications for clinical practice. The WOSCOPS Study Group. Eur Heart J 1996;17:1634 . 
[37] Burnet DL, Elliott LD, Quinn MT, Plaut AJ, Schwartz MA, Chin MH. Preventing diabetes in the clinical setting. J Gen Intern Med 2006;21:84- 93. 